\title{
O papel do cuidado com as Doenças Crônicas não Transmissíveis na Atenção Primária em Saúde: um olhar da Antropologia da Saúde
}

The role of care for Chronic Non-Communicable Diseases in Primary Health Care: a look from the Anthropology of Health

El papel de la atención de las Enfermedades Crónicas no Transmisibles en la Atención Primaria de Salud: una mirada desde la Antropología de la Salud

\section{Resumo}

Introdução: Os paradigmas atuais na antropologia da saúde procuram ressaltar aspectos como a interação e a relacionalidade inerentes às práticas sociais na saúde, através do cuidado. As doenças crônicas não transmissíveis (DCNT) são as principais causas de morte e incapacidade em todo o mundo. Busca-se, nesse ensaio, apresentar uma reflexão sobre as sobre as contribuições da antropologia da saúde e da doença para a construção de novas práticas em saúde. Metodologia: Esse ensaio foi produzido a partir dos debates e referencial bibliográfico trabalhado em sala de aula durante a disciplina de Seminários de Ciências Sociais, do Programa de Pós-Graduação em Saúde Coletiva, da Universidade Federal de Santa Catarina (UFSC). Considerações finais: O crescimento em nível global da incidência coloca em alerta a saúde e o cuidado prestado na Atenção Primária a Saúde (APS) a essas doenças. O enfoque dado dentro do cuidado baseia-se nas estratégias preventivas baseadas na mudança de comportamento dos indivíduos, o que por muitas vezes falha pela falta de adesão a questão terapêutica.

Palavras-chave: Doenças crônicas não transmissíveis; Antropologia da saúde; Cuidado; Processo saúde doença; Atenção Primária a Saúde (APS).

\begin{abstract}
Introduction: Current paradigms in health anthropology seek to emphasize aspects such as interaction and relationality inherent to social practices in health, through care. Chronic Non-Communicable Diseases (NCDs) are the leading causes of death and disability worldwide. This essay seeks to present a reflection on the contributions of the anthropology of health and disease to the construction of new health practices. Methodology: This essay was produced from debates and bibliographic references worked in the classroom during the course of Social Science Seminars, of the Graduate Program in Public Health, at the Federal University of Santa Catarina (UFSC). Final considerations: The global increase in incidence raises awareness of health and the care provided in Primary Health Care (PHC) to these diseases. The focus given within care is based on preventive strategies based on changing the behavior of individuals, which often fails due to the lack of adherence to the therapeutic issue.
\end{abstract}

Keywords: Chronic non-communicable diseases; Anthropology of health; Caution; Health disease process; Primary Health Care (PHC).

\section{Resumen}

Introducción: Los paradigmas actuales en antropología de la salud buscan enfatizar aspectos como la interacción y la relacionalidad inherentes a las prácticas sociales en salud, a través del cuidado. Las enfermedades crónicas no 
transmisibles (ENT) son las principales causas de muerte y discapacidad en todo el mundo. Este ensayo busca presentar una reflexión sobre los aportes de la antropología de la salud y la enfermedad a la construcción de nuevas prácticas de salud. Metodología: Este ensayo fue elaborado a partir de debates y referencias bibliográficas trabajadas en el aula durante el curso de Seminarios de Ciencias Sociales, del Programa de Posgrado en Salud Pública, de la Universidad Federal de Santa Catarina (UFSC). Consideraciones finales: El aumento global de la incidencia sensibiliza sobre la salud y la atención que se brinda en Atención Primaria de Salud (APS) a estas enfermedades. El enfoque que se da dentro de la atención se basa en estrategias preventivas basadas en el cambio de comportamiento de los individuos, que muchas veces fracasa por la falta de adherencia al tema terapéutico.

Palabras clave: Enfermedades crónicas no transmisibles; Antropología de la salud; Precaución; Proceso de enfermedad de salud; Atención Primaria de Salud (APS).

\section{Introdução}

As doenças crônicas não transmissíveis (DCNT) se caracterizam por um conjunto de patologias de múltiplas causas e fatores de risco, longos períodos de latência e curso prolongado, origem infecciosa ou não, podendo resultar em incapacidades funcionais (Brasil, 2008).

No início do século XX, as doenças infecciosas eram as principais causas de óbito na população mundial, atualmente, as DCNT se constituem como as principais causas de mortalidade, resultado das melhores condições socioeconômicas de saúde nas últimas décadas (Figueiredo, Ceccon, \& Figueiredo, 2021).

No Brasil, as DCNT representam a principal carga de doenças e mortes na população, constituindo-se como um importante problema de saúde pública, por atingir indivíduos de todas as camadas socioeconômicas, mas, principalmente aqueles com maiores graus de vulnerabilidades (Malta, Andrade, Oliveira, Moura, Prado, \& Souza, 2019; Malta, \& Freitas, 2020; Figueiredo, Ceccon, \& Figueiredo, 2021). Para tanto um ponto importante no combate das DCNT baseia-se nas estratégias para mudança de estilos de vida na população em geral, visando redução da prevalência de fatores de risco para doenças crônicas não transmissíveis (DCNT), têm sido discutidas e incentivadas em vários países (Capilheira, \& Santos, 2011).

Diversas intervenções para reduzir o tabagismo, promover atividade física e consumo de alimentos saudáveis foram realizadas, mas suas avaliações demonstraram resultados discretos, o que implica a adesão (OPAS, 2003; Capilheira, \& Santos, 2011). A extensa cadeia causal desde a implantação das intervenções até o impacto, o funcionamento do sistema de saúde (acesso, disponibilidade de exames complementares e medicamentos), o meio ambiente e as características sociodemográficas, culturais e epidemiológicas da população estão entre as razões da baixa efetividade (Santos, \& Victora, 2004).

Um ponto importante referido por Capilheira e Santos (2011) é o envolvimento dos serviços de saúde como uma estratégia importante para o sucesso e sustentabilidade das intervenções preventivas em DCNT. Como forma de auxiliar a construção de estratégias para se alcançar resultados nessas ações preventivas é importante o conhecimento da realidade local, levando-se em conta construção que a pessoa compreende como saúde e doença, e como a sua ação/ adesão influencia no processo.

A medida da efetividade de um programa de saúde depende da extensão em que a população que é coberta pelo mesmo aceita, utiliza e participa, essa efetividade parece, assim, ser dependente do conhecimento prévio das maneiras características de pensar e agir associadas à saúde nessa população e da habilidade do programa em integrar esse conhecimento. Para compreender essa constante, é necessário buscar luz na influência do contexto social e cultural sobre "as maneiras de pensar e de agir” das populações frente aos seus problemas de saúde e, a partir daí, situar a contribuição específica da abordagem antropológica. A Antropologia Médica se apresenta como uma perspectiva complementar e enriquecedora para a abordagem dos problemas de saúde pública e das doenças crônicas não transmissíveis (Uchôa, \& Vidal, 1994).

A compreensão do cuidado na Atenção Primária em Saúde com as DCNT tem um envolvimento na estratégia proposta do envolvimento dos serviços e profissionais de saúde como uma ferramenta efetiva e sustentável de intervenção nas 
comunidades, especialmente na prevenção pelo combate aos seus fatores de risco. A abordagem tradicional concernente ao estilo de vida tem se estruturado em aumentar o conhecimento e modificar o modo de pensar dos indivíduos, imputando sobre eles, assim, a carga da mudança comportamental. Por outro lado, grandes mudanças na população, em todos os níveis, exigem um consenso entre as várias partes interessadas, que trazem suas próprias perspectivas para tratar as questões em foco. Isso é importante na esfera política, em que os interesses públicos e privados são mais evidentes (como no caso do controle do tabaco). Mas, também é importante na comunidade, onde a estrutura de poder exerce influência sobre as possibilidades de atuação de diferentes participantes. A prestação de serviço de saúde, em particular o atendimento ambulatorial preventivo, não está isento. Em geral, exige-se mudança na estrutura organizacional dos serviços de saúde para que os indivíduos em risco e/ou com a doença tenham uma participação ativa para que as mudanças comportamentais realmente aconteçam (OPAS, 2003).

Deve-se conhecer o perfil dos profissionais desse nível de atenção e sua atuação junto aos usuários, a fim de que se planejem estratégias de combate às DCNT adequadas à realidade de cada local. Sendo os recursos humanos elementos centrais da qualidade do cuidado à saúde oferecido à população, o atual estudo consistiu em uma avaliação da estrutura (características dos recursos humanos) e processo de atenção nas unidades básicas de saúde (UBS). Este ensaio busca revisitar a perspectiva das Doenças Crônicas não Transmissíveis sob a ótica da Antropologia Médica.

\section{Saúde e doença: as doenças crônicas não transmissíveis (DCNT)}

As doenças crônicas não transmissíveis (DCNT) incluem as doenças do aparelho cardiovascular, diabetes mellitus (DM), doenças de origem respiratória e os cânceres, constituindo a maior carga de morbimortalidade no mundo e sendo responsáveis por $63 \%$ das mortes globais. Essas doenças acarretam perda de qualidade de vida, limitações e incapacidades1,2. As mortes por DCNT afetam predominantemente os países em desenvolvimento, nos quais cerca de um terço dos óbitos ocorrem em pessoas com menos de 60 anos de idade, enquanto nos países desenvolvidos a mortalidade prematura (faixa etária de 30 a 69 anos) corresponde a menos de 13\% dos casos (WHO, 2011; Malta, Andrade, Oliveira, Moura, Prado, \& Souza, 2019).

As DCNT atingem indivíduos de todas as camadas socioeconômicas e, de forma mais intensa, aqueles pertencentes a grupos vulneráveis, como os de baixa escolaridade e renda, aumentando ainda mais a pobreza dos que são acometidos pelas incapacidades, limitações e redução da força produtiva (Agebunde, Mathers, Adam, Ortegon, \& Strong, 2007; Bonita, Magnusson, Bovet, Zhao, Malta, Geneau, et al., 2013; Malta, Andrade, Oliveira, Moura, Prado, \& Souza, 2019)

Em 2011, foi assinada uma Declaração de Alto Nível na Organização das Nações Unidas com a missão de reduzir as taxas de mortalidade por DCNT. Em 2013, a Assembleia Mundial da Saúde aprovou o Plano de Ação Global de DCNT, estabelecendo o compromisso dos países membros com a redução da probabilidade de morte por DCNT em 25\% entre 2015 e 2025.

A OMS divulgou um conjunto de evidências que apontam a importância das ações de promoção à saúde, implementando políticas públicas intra e intersetoriais que facilitem práticas saudáveis, como alimentação adequada, redução do sal nos alimentos, espaços públicos para apoiar a atividade física, ambientes livres de fumo, regulamentação da propaganda de álcool e outras (ONU, 2011). Além disso, cabe o investimento na atenção básica e no acesso às tecnologias de média e alta complexidade, quando necessário, visando ao cuidado integral dos portadores de DCNT. Essas doenças têm curso prolongado e requerem abordagem longitudinal, integral, com investimento no autocuidado e no vínculo. Essencialmente, deve-se atuar reduzindo iniquidades em saúde e garantindo acesso aos cuidados a toda a população, em especial aos grupos mais vulneráveis, dada a maior concentração das DCNT e seus fatores de risco na população de baixa renda e escolaridade (ONU, 2011; Malta, Geneau, et al., 2013; Malta, Andrade, Oliveira, Moura, Prado, \& Souza, 2019).

Ao se analisar os programas de saúde que dão suporte a terapêutica das DCNT, há a necessidade de compreender a 
concepção de saúde e doença, a qual permite compreender a adesão e construção do entendimento da população, portanto, trata-se de um problema de saúde pública de alta relevância, e sua abrangência tem levado ao questionamento sobre medidas preventivas, promotoras de saúde e de vigilância epidemiológica, bem como sobre o investimento de políticas públicas (Minayo, \& Gualhano, 2015; Ferreira, 2020). Do ponto de vista socioantropológico, as DCNT são exemplares na ilustração da experiência do mundo do doente e de sua interação com os profissionais da saúde. O universo das DCNT toma conta de todas as dimensões da vida do enfermo, tanto em seus aspectos simbólicos como socioeconômicos (Ferreira, J., 2020).

$\mathrm{Na}$ abordagem sociológica, os problemas de saúde são apreendidos em sua dimensão social e não individual. A sociologia da saúde investiga a determinação que exercem os contextos social e institucional sobre as enfermidades e os comportamentos delas decorrentes (Uchôa, \& Vidal, 1994).

Na perspectiva antropológica, o universo sociocultural do doente é visto não mais como obstáculo maior à efetividade dos programas e práticas terapêuticas, mas como o contexto onde se enraízam as concepções sobre as doenças, as explicações fornecidas e os comportamentos diante delas (Uchôa, \& Vidal, 1994).

A antropologia considera que a saúde e o que se relaciona a ela (conhecimento do risco, ideias sobre prevenção, noções sobre causalidade, ideias sobre tratamentos apropriados, etc.) são fenômenos culturalmente construídos e culturalmente interpretados (Nichter, 1989). Com isso, a perspectiva qualitativa é empregada para identificar e analisar a mediação que exercem os fatores sociais e culturais na construção de formas características de pensar e agir frente à saúde e à doença. Integrando uma apreensão da dimensão cultural, a antropologia médica vem, ao lado da sociologia da saúde e da epidemiologia, contribuir para ampliar o contexto que deve ser levado em consideração na leitura dos processos patológicos (Uchôa, \& Vidal, 1994; Teixeira, 2017; Tavares, 2017; Gerhardt, 2019; Santos, Maciel, Flores, \& Ferreira, 2019).

\section{Cuidado das DNCT na Atenção Primária à Saúde (APS) e Antropologia da Saúde}

Apesar da biomedicina ainda se justificar como saber hegemônico, foi com o fortalecimento da antropologia da saúde e da doença nas últimas décadas no Brasil que se passou a defender um relativismo relacionado ao processo saúde/doença e às práticas de saúde, onde os saberes e práticas de qualquer sistema médico são percebidos enquanto construções socioculturais (Becker, Rosa, Manfrini, Backes, Meirelles, \& Santos, 2009).

Em outras palavras, o fenômeno saúde doença não pode ser entendido à luz unicamente de instrumentos anátomo fisiológicos da medicina (Minayo, 1991), mas deve considerar a visão de mundo dos diferentes segmentos da sociedade, bem como suas crenças e cultura. Significa dizer que nenhum ser humano deve ser observado apenas pelo lado biológico, mas percebido em seu contexto sociocultural (Santos, Silva, Sampaio, Sena, Gomes, \& Lima, 2011)

Tendo em vista que boa parte dos profissionais de saúde nos dias de hoje - seja pela dinâmica nos serviços de saúde, seja pela falta de capacitação ou mesmo pelo descaso com o usuário - ainda reproduzem um ideal positivista, impondo um modelo teórico fechado, onde o usuário do serviço não participa ativamente do processo, além de dissociar a saúde e a doença dos aspectos e dimensões históricas, sociais e culturais dos indivíduos (Santos, Silva, Sampaio, Sena, Gomes, \& Lima, 2011).

De modo geral para Uchôa e Vidal (1994), os programas de saúde partem do pressuposto de que a informação gera uma transformação automática dos comportamentos das populações frente às doenças. Essa abordagem negligencia os diferentes fatores sociais e culturais que intervêm na adoção desses comportamentos, os autores relatam a grande influência que exercem a semiologia popular e as concepções culturais de causalidade sobre os comportamentos adotados frente às doenças (Souza, Guimarães, \& Silva, 2017; Nilo, 2018; Romero, Castanheira, Marques, Muzy, Sabbadini, \& Silva, 2018; Vieira-da-Silva, \& Furtado, 2020).

No contexto do cuidado, nota-se que muitos profissionais não consideram as informações culturais, considerando-as como irrelevantes para as intervenções preventivas e terapêuticas na área da saúde. Em geral, são tidas como essenciais 
unicamente aqueles referentes ao diagnóstico biomédico (Uchôa, \& Vidal, 1994).

Para Uchôa e Vidal (1994), vários estudos revelam que os comportamentos de uma população frente a seus problemas de saúde, incluindo a utilização dos serviços médicos disponíveis, são construídos a partir de universos socioculturais específicos. Eles apontam a necessidade de enraizarem-se os programas de educação e o planejamento em saúde em conhecimento prévio das formas características de pensar e agir predominantes nas populações junto às quais se quer intervir (Hielscher, \& Sommerfeld, 1985; Nyamwaya, 1987; Nichter, 1989; Corin, Uchôa, Bebeau, \& Harnois, 1989; Fincham, 1992; Agyepong, 1992; Green, 1992; Larrubia, Silva, \& Freitas, 2015).

O dualismo cartesiano entre corpo e espírito seria o precursor imediato das concepções biomédicas contemporâneas de organismo humano e do pensamento materialista radical que caracteriza a biomedicina (Scheper-Hugues, \& Lock, 1987; Teixeira, 2017; Tavares, 2017; Gerhardt, 2019; Santos, Maciel, Flores, \& Ferreira, 2019). A doença é ora vista como um problema físico ou mental, ora como biológico ou psicossocial, mas raramente como fenômeno multidimensional. A fragmentação do objeto gera a fragmentação das abordagens. A descontinuidade entre as diferentes abordagens retarda a apreensão multidimensional do objeto. A contribuição da antropologia é aqui extremamente importante. Ela ressitua nossas premissas básicas no horizonte epistemológico ocidental, tornando possível uma perspectiva crítica frente a nossas "verdades" mais fundamentais e favorecendo a construção de um novo paradigma para a abordagem da saúde e da doença (Uchôa, \& Vidal, 1994; Larrubia, Silva, \& Freitas, 2015).

As ciências antropológicas e sociais têm proposto uma concepção construcionista da doença, das estratégias de atenção, mas também da vida cotidiana onde se processa o padecimento, o que, entre outras razões, a têm conduzido observar historicamente o papel da biomedicina com respeito a toda uma diversidade de problemas. Se bem que o processo de medicalização e, em especial, o da "psiquiatrização" têm sido os mais investigados, esta abordagem tem sido aplicada a temas tão diversos como o autocuidado ou o processo de alcoolização. Para além dos tipos de interpretações específicas resultantes dessas investigações, as mesmas têm posto em relevo o fato de que ditos processos só podem ser realmente compreendidos a partir de uma perspectiva diacrônica construcionista e têm evidenciado a significação paradoxal da biomedicina tanto na construção técnica da enfermidade (disease) como também na construção social do padecimento (illness). (Menéndez, 1998; Larrubia, Silva, \& Freitas, 2015).

A Antropologia Médica e também outras disciplinas sócio-históricas tem produzido materiais empíricos e teóricos que supõem não só contribuições, senão também possibilidades de articulação com a perspectiva epidemiológica, sempre e quando exista realmente um interesse de articulação e no prime a exclusão nos fatos. A seguir enumeraremos algumas destas contribuições socioantropológicas (Menéndez, 1998).

A mudança para primeiro plano das enfermidades crônico-degenerativas também favoreceu o reconhecimento das técnicas qualitativas, dado a crescente importância outorgada aos estilos de vida tanto na causação como para o controle e, sobretudo, autocontrole destas doenças. A recente ênfase dada a experiência do sujeito enfermo, a convivência com "seu" padecimento e o "autocuidado" como expressões que podem assegurar uma maior esperança e qualidade de vida, reforçam ainda mais a significação das técnicas qualitativas. Estas últimas referências nos conduzem a outro fator que tem dado impulso ao desenvolvimento da abordagem qualitativa (Menéndez, 1998).

As propostas de Atenção Primária, não só as de Atenção Primária Integral e Seletiva, mas também as de Atenção Médica Primária, supõem a inclusão de atividades de participação social e organização comunitária, a utilização de práticas populares e o impulso da educação para a saúde. Menéndez (1998) refere que a maioria da informação a ser obtida para dar impulso a estas estratégias refere-se a processos sociais, culturais, ideológicos e políticos e que, além disso, é requerido incluir a ação a partir não apenas dos serviços de saúde, senão, sobretudo, a partir da própria população.

A antropologia desenvolveu importante aparelhagem conceitual e metodológica para o estudo sistemático das 
maneiras culturais de pensar e de agir associadas à saúde. Ela permite examinar as relações (interações e contradições) entre os modelos de prática, que suportam a organização dos serviços, os programas de prevenção e as intervenções terapêuticas, e os modelos culturais dos usuários. A partir daí, ela fornece parâmetros para a reformulação da questão da adequação sociocultural dos diferentes programas de saúde (Uchôa, \& Vidal, 1994).

\section{Considerações Finais}

A perspectiva da saúde enquanto construção sociocultural, e não biológica, permite uma análise crítica de três aspectos do processo de saúde/doença que são relevantes para as políticas e os serviços em saúde: práticas terapêuticas, especialistas em cura e a emergência de novas formas de atenção; dinâmicas envolvidas nas práticas de autoatenção em contextos etnográficos específicos; e relações entre biomedicina e práticas locais (Becker, Rosa, Manfrini, Backes, Meirelles, \& Santos, 2009; Langdon, 2014).

Para tanto, é importante reforçar o cuidado as doenças crônicas não transmissíveis como uma prática consciente de educação para a saúde, dentro do contexto da Atenção Primária a Saúde (APS), e cabe aos profissionais da saúde, investigar os hábitos de vida e aspectos sociais de cada paciente, além de conhecer as medidas preventivas específicas, o custo de suas aplicações e a reação dos pacientes. Conhecer a cultura local e os hábitos são fatores que implicam no sucesso do tratamento.

\section{Referências}

Agebunde, D. O., Mathers, C. D., Adam, T., Ortegon, M. \& Strong, K. (2007). The burden and costs of chronic diseases in low-income Middle-income countries. Lancet., 370(9603), 1929-1938.

Agyepong, I. A. (1992). Malaria ethnomedical perception and pratice in na Adangbe farming Community and implications for control. Soc Sci Med, 35(2), 131-137.

Becker, S. G., Rosa, L. M., Manfrini, G. C., Backes, T. T. S., Meirelles, B. H. S. \& Santos, S. M. A. (2009). Dialogando sobre o processo saúde/doença com a Antropologia: entrevista com Esther Jean Langdon. Rev. Bras. Enferm., 62(2), 1-4.

Bonita, R., Magnusson, R., Bovet, L., Zhao, D., Malta, D. C., Geneau, R. et al. (2013). Country actions to meet um commitmentes on non-comunicable diseases: a stepwise approach. Lancet., 381(9866), 575-574.

Brasil. (2008). Diretrizes e recomendações para o cuidado integral de doenças crônicas não transmissíveis: promoção da saúde, vigilância, prevenção e assistência. Recuperado de https://bvsms.saude.gov.br/bvs/publicacoes/diretrizes_recomendacoes_cuidado_doencas_cronicas.pdf.

Capilheira, M. \& Santos, I. S. (2011). Doenças crônicas não transmissíveis: desempenho no cuidado médico em atenção primária à saúde no sul do Brasil. Cad. Saúde Pública, 27(6), 1143-1153.

Corin, E., Uchôa, E., Bebeau, G. \& Harnois, G. (1989). Les Attitudes Dans le Champ de la Santé Mentale. Repères Théoriques et Méthodologiques pour une Étude Ethnographique et Comparative. Rapport Technique. Montreal. Centre de Recherche de l'Hôpital Douglas, Centre Collaborateur OMS.

Ferreira, J. (2020). Doenças crônicas não transmissíveis e os dilemas do cuidado: a teoria da ordem negociada revisitada. Saúde Soc. São Paulo, 29(4):e109149, 1-9

Figueiredo, A. E. B., Ceccon, R. F. \& Figueiredo, J. H. C. (2021). Doenças crônicas não transmissíveis e suas implicações na vida de idosos dependentes. Ciênc. Saúde Colet., 26(1), 77-88.

Fincham, S. (1992). Community health promotion programs. Soc Sci Med., 35, 239-249.

Gerhardt, T. E. (2019). Cultura e cuidado: dilemas e desafios do ensino na antropologia na graduação em Saúde Coletiva. Saude soc., 28(2), 1-15.

Green, E. C. (1992). Sexually transmited disease, ethnomedicine anda health policy in Africa. Soc Sci Med., 35, 121-130.

Hielscher, S. \& Sommerfeld, J. (1985). Concepts of illness and the utilisation of health care services in a rural Malien Village. Soc Sci Med., 21(4), 469-481.

Langdon, E. J. (2014). Os diálogos da Antropologia com a saúde: contribuições para as políticas públicas. Ciênc. saúde coletiva, 19(4), 1019-1029.

Larrubia, B. C., Silva, N. E., Jr. \& Freitas, I. M. (2015). Antropologia da saúde e doença: contribuições para os serviços públicos de saúde. Revista Científica Multidisciplinar Núcleo do Conhecimento, 04(08), 05-28.

Malta, D. C. \& Freitas, M. I. F. (2020). Doenças crônicas não transmissíveis e fatores de risco e proteção em adultos com ou sem plano de saúde. Ciênc. saúde coletiva, 25(8), 2973-2984.

Malta, D. C., Andrade, S. S. C. A., Oliveira, T. P., Moura, L., Prado, R. R. \& Souza, M. F. M. (2019). Probabilidade de morte prematura por doenças crônicas não transmissíveis, Brasil e regiões, projeções para 2025. Rev. bras. epidemiol., 22:190030, 1-13. 
Research, Society and Development, v. 10, n. 12, e267101220250, 2021

(CC BY 4.0) | ISSN 2525-3409 | DOI: http://dx.doi.org/10.33448/rsd-v10i12.20250

Menéndez, E. (1998). Antropologia médica e epidemiologia: processo de convergência ou processo de medicalização? In: Alves, P.C. \& Rabelo, M. C. (1998). Antropologia da saúde: traçando identidade e explorando fronteiras. Rio de Janeiro: Relume Dumará.

Minayo, C. C. S. \& Gualhano, L. (2015). Vigilância de doenças crônicas e violências, o desafio contemporâneo. Ciência e Saúde Coletiva, 20(3), 1-3.

Minayo, M. C. S. (1991). Abordagem antropológica para avaliação de políticas sociais. Rev. Saúde Pública, 25(3), 1-6.

Nichter, M. (1989). Anthropology and International health: South Asian case Studies. Heidelberg, Germany. Springer Netherlands.

Nilo, M. C. B. G. (2018). Análise de Rede Social como estratégia para avaliação de programas de saúde para o controle da tuberculose. REDES - Revista Hispana para el Análisis de Redes Sociales, 29(2), 237-247.

Nyamwaya, D. (1987). A case study of interactions netween indigenous and western medicine among the Pokot of Kenya. Soc Sci Med., 25(12), $1277-1287$.

Organização das Nações Unidas (ONU). (2011). Political declaration of the high-level meeting of the general assembly on the prevention and control of noncommunicable diseases: sixtth ssession: agenda item 117. Recuperado de https://digitallibrary.un.org/record/710899.

Organização Pan-Americana de Saúde (OPAS. (2003). CARMEN: iniciativa para a prevenção de doenças crônicas não transmissíveis nas Américas. Recuperado de https://apsredes.org/gestores-das-americas-debatem-doencas-cronicas/.

Romero, D. E., Castanheira, D., Marques, A. P., Muzy, J., Sabbadini, L. \& Silva, R. S. (2018). Metodologia integrada de acompanhamento de políticas públicas e situação de saúde: o SISAP-Idoso. Ciênc. saúde colet., 23(8), 2641-2650.

Santos, A. C. B, Silva, A. F., Sampaio, D. L., Sena, L. X., Gomes, V. R. \& Lima, V. L. (2012). Antropologia da saúde e da doença: contribuições para a construção de novas práticas em saúde. Rev. NUFEN, 4(2), 11-20.

Santos, H. L. P. C., Maciel, F. B. M., Flores, R. E. L. \& Ferreira, P. R. S. (2019). Antropologia e saúde caminhos possíveis para (re)pensar a prática médica. REVASF, 9(20), 50-64.

Santos, I. S. \& Victora, C. G. (2004). Serviços de saúde: epidemiologia, pesquisa e avaliação. Cad Saúde Pública, 20(Sup 2), 337-341.

Scheper-Hugues, N. \& Lock, M. M. (1987). The mindful body: a prolegomenon to Future Work in Medical Anthropology. JSTOR, 1(1), 6-41.

Souza, E. C., Guimarães, J. M. X. \& Silva, M. R. F. (2017). Estudos de avaliabilidade de políticas e programas de saúde no Brasil: revisão integrativa. SANARE, 16(2), 85-92.

Tavares, F. (2017). Rediscutindo conceitos na antropologia da saúde: notas sobre os agenciamentos terapêuticos. Mana, 23(1), 201-228.

Teixeira, M. Z. (2017). Antropologia médica vitalista: uma ampliação ao entendimento do processo de adoecimento humano. Revista de Medicina, 96(3), 145158.

Uchôa, E. \& Vidal, J. M. (1994). Antropologia médica: elementos conceituais e metodológicos para uma abordagem de saúde e da doença. Cad. Saúde Pública, 10(4), 597-504.

Vieira-da-Silva, L. M. \& Furtado, J. P. (2020). A avaliação de programas de saúde: continuidades e mudanças. Cad. Saúde Pública, 36(9):e00237219, 1-8. 